\title{
Editorials
}

\section{Migraine management:}

\author{
beyond pills
}

\section{INTRODUCTION}

Migraine is the second most common type of primary headache disorder and affects approximately 15\% of the global population. Although often regarded as a benign neurological condition, migraine is associated with significant morbidity and reduced quality of life in some patients. In 2015 the Global Burden of Disease Study showed that migraine was the seventh highest cause worldwide of years lost to disability. Migraine is characterised by episodic, moderately-severe headache, which lasts for at least 4 hours and is typically associated with nausea, vomiting, photophobia, phonaphobia, and is exacerbated with exercise. During severe attacks patients often find it difficult to function, which can lead to significant problems at home and in the workplace. A diagnosis of chronic migraine can be made in patients who, on average, have $>15$ days of headache a month, for $\geq 3$ months, of which on at least 8 days a month there are features of migraine. There is no cure for migraine, but positive changes to lifestyle and the use of migraine preventives can significantly reduce the frequency and severity of attacks. In some patients migraine preventives either fail or are not tolerated. In order to function such patients often resort to liberal use of simple analgesics, which over time may lead to the development of medicationoveruse headache. A high proportion of patients referred to neurology clinics report that they do not like taking daily oral medication, which is either poorly tolerated or ineffective.

Over the past 5 years there have been significant developments in the management of migraine and particularly chronic migraine. This editorial will examine the strategies that are typically adopted in a specialist headache clinic and discuss the latest treatment options, including, CBT and mindfulness training, acupuncture, the use of botulinum toxin, electrical nerve stimulation, occipital nerve blocks, and therapies that target the calcitonin generelated peptide (CGRP) pathway.

\section{COGNITIVE BEHAVIOURAL THERAPY AND MINDFULNESS}

Cognitive behavioural therapy (CBT), including the use of stress management,

"In 2015 the Global Burden of Disease Study showed that migraine was the seventh highest cause worldwide of years lost to disability.

relaxation, and biofeedback techniques is used to address the dysfunctional thinking which often accompanies pain and leads to psychological symptoms such as depression, anxiety, and sleep disturbance. As well as reducing psychological symptoms, some patients receiving CBT also report less pain during migraine attacks. $^{2}$

Mindfulness training has become increasingly popular over the past 5 years, is more readily accessible than traditional CBT, and can often be trialled without the need of a therapist. 'Mindfulness' has many definitions, but in our opinion is best described by the Mayo clinic as .... the act of being intensely aware of what you're sensing and feeling at every moment without interpretation or judgement."

Preliminary data from one ongoing study in patients with chronic migraine complicated by medication-overuse headache, ${ }^{4}$ indicated that patients who received mindfulness training had a similar reduction in headache frequency compared to those who received migraine preventives. Furthermore, a higher proportion of patients receiving mindfulness training showed evidence of significant clinical improvement (defined as reduction of $\geq 50 \%$ ) and consumed fewer acute medications than those in the pharmacotherapy group. Although not for everyone, in our experience, patients with headache receiving mindfulness training often report feeling more connected to their bodies and more in control of their symptoms. Mindfulness training can be delivered in a variety of ways including group setting, one-to-one, or through an online course or self-help books.

\section{ACUPUNCTURE}

Many patients who have failed on more traditional migraine prophylaxis turn to acupuncture. The science behind acupuncture and its observed benefit in some patients with migraine is constantly under evaluation. A recent Cochrane Review concluded that acupuncture reduced the frequency of attacks in episodic migraine and '... may be at least similarly effective as treatment with prophylactic drugs. ${ }^{5}$

Although considered by some as nothing more than a placebo, ${ }^{6}$ some patients undoubtedly benefit and it is recommended by the National Institute for Health and Care Excellence (NICE) as a second-line therapy in the treatment of migraine. Some GPs still offer acupuncture, but many patients pay privately to see therapists. When recommending therapy we always direct our patients to www.acupuncture.org. uk so that they can find a locally-accredited therapist.

\section{BOTULINUM TOXIN}

There is now a significant body of evidence that demonstrates that botulinum toxin is an effective prophylactive treatment for patients with chronic migraine. According to the original trial, patients should receive $31 \times 5$-unit subcutaneous injections of botulinum toxin (Botox) in a set pattern over the forehead, scalp, neck, and shoulders at 3-monthly intervals. ${ }^{7}$ Although this trial only showed a modest reduction in headache days per month compared to placebo $(-8.4$ versus -6.6 , respectively), in clinical practice treatment with botulinum toxin appears to be very effective in the majority of patients. In 2012, botulinum toxin was approved by NICE for the treatment of chronic migraine and there are now a number of centres across the UK that offers this as part of their headache service. Starting criteria stipulate that patients should suffer chronic migraine and have already failed on three migraine preventives.

\section{NON-INVASIVE NEUROSTIMULATION}

There is growing interest in neuromodulation and the use of electronic devices to treat patients with migraine and cluster headache.

Non-invasive nerve stimulation with 
the gammaCore ${ }^{\circledR}$ (electroCore) device has been shown to be safe and effective in the treatment of acute cluster headache $e^{8}$ and has the potential to reduce triptan use. Electrical stimulation of the vagus nerve is achieved by pressing the gammaCore device against the side of the neck and in the case of cluster headache this should be ipsilateral to pain. Although the exact mechanism of action remains unknown, modulation of trigeminal pain pathways has been implicated. The effectiveness of gammaCore in patients with migraine is less clear and although trials are still ongoing we have found it very effective in some patients. Transcutaneous stimulation of the supraorbital nerve with the Cefaly ${ }^{\circledR}$ (Cefaly Technology) device has been shown to be beneficial in reducing headache days in patients with frequent episodic migraine, ${ }^{9}$ but not in management of acute migraine or cluster headache. The third of these devices is the eNeura ${ }^{\circledR}$ (eNeura Inc) transcranial magnet. This is placed over the back of the head and used to deliver a single pulse of transcranial magnetic stimulation during migraine attacks. This in turn is thought to disrupt spreading cortical depression, which is important in the pathogenesis of migraine aura and pain. There is some evidence that the eNeura is useful in the treatment of acute migraine attacks with aura ${ }^{10}$ and may also be effective in migraine prevention.

Use of gammaCore and eNeura devices currently require authorisation by a clinician specialising in headache and are billed monthly according to use. The Cefaly can be purchased as a one-off by the patient and does not require authorisation.

\section{GREATER OCCIPITAL NERVE BLOCKS}

Greater occipital nerve blocks (GONBs) are frequently used in the management of patients with headache, especially in those with occipital neuralgia and cervicogenic headache. The procedure involves injection of a local anaesthetic (for example, 2\% lidocaine), with or without steroid (for example, depomedronel over one or both greater occipital nerves. This is well tolerated and often administered in the headache clinic. A recent study showed that bilateral GONBs can be useful in the management of patients with chronic migraine $^{11}$ and in our experience may offer an alternative to patients who fail with botulinum toxin. GONBs can also be useful in patients with cluster headache ${ }^{12}$ especially early in the cluster bout and may give a few days of pain relief during the titration of other prophylactic drugs.
Other pericranial nerve blocks can also be administered, but require more expertise and are less readily available.

\section{CGRP RECEPTOR ANTAGONISTS AND ANTIBODIES AGAINST CGRP}

In recent months there has been considerable excitement in the news media about a novel wave of therapies which target CGRP, an important molecule which has been implicated in the pathophysiology of migraine. Monoclonal antibodies against CGRP and the CGRP receptor have been shown to be well tolerated, safe, and effective in migraine prevention..$^{13}$ Due to the long half-life of antibodies, dosing is likely to be monthly and given as a subcutaneous injection or intravenous infusion. However, the cost of these new treatments is likely to be high and will need to be carefully balanced against their effectiveness and benefits of improved quality of life.

\section{CONCLUSION}

The management of pill-resistant migraine can be soul destroying for patients and frustrating for treating physicians and often leads to the development of medicationoveruse headache. In light of recent trial data supporting the use of botulinum toxin various electronic devices, and nerve blocks, we suggest that GPs and neurologists look 'beyond the pills' in patients who do not respond to conventional migraine prophylaxis. For patients who want to avoid medication altogether, there is some evidence that CBT, mindfulness training, and acupuncture are helpful. With the advent of drugs that target CGRP there will be additional options for migraineurs who have failed on other types of prophylaxis.

\section{Benjamin R Wakerley,}

Consultant neurologist, Gloucestershire Royal Hospital, Gloucester.

\section{Sarah Davies,}

GP, Gloucestershire Royal Hospital, Gloucester, and Chipping Campden Surgery, Back Ends, Chipping Campden, Gloucestershire.

\section{Provenance}

Freely submitted; externally peer reviewed.

\section{Competing interests}

Benjamin R Wakerly has received an educational grant from Novartis in the past. Sarah Davis has declared no competing interests.

DOI: https://doi.org/10.3399/bjgp18X698969

\section{ADDRESS FOR CORRESPONDENCE}

Benjamin R Wakerley

Department of Neurology, Gloucestershire Royal

Hospital, Gloucester GL1 3NN, UK.

Email: benwakerleyðfastmail.fm

\section{REFERENCES}

1. GBD 2015 Disease and Injury Incidence and Prevalence Collaborators. Global, regional, and national incidence, prevalence, and years lived with disability for 310 diseases and injuries, 1990-2015: a systematic analysis for the Global Burden of Disease Study 2015. Lancet 2016; 388(10053): 1545-1602.

2. Harris $P$, Loveman $E$, Clegg A, et al. Systematic review of cognitive behavioural therapy for the management of headaches and migraines in adults. Br J Pain 2015; 9: 213-224.

3. Dorfner M.Use mindfulness to improve wellbeing. 2015. https://newsnetwork.mayoclinic. org/discussion/use-mindfulness-to-improvewell-being/ (accessed 6 Sep 2018)

4. Andrasik F, Grazzi L, D’Amico D, et al. Mindfulness and headache: $A$ 'new' old treatment, with new findings. Cephalalgia 2016; 36(12): 1192-1205.

5. Linde $\mathrm{K}$, Allais $\mathrm{G}$, Brinkhaus $\mathrm{B}$, et al. Acupuncture for the prevention of episodic migraine. Cochrane Database Syst Rev 2016; 6: CD001218.

6. Solomon S. Acupuncture for headache. It's still all placebo. Headache 2017; 57(1): 143-146.

7. Dodick DW, Turkel CC, DeGryse RE, et al. OnabotulinumtoxinA for treatment of chronic migraine: pooled results from the double-blind, randomized, placebo-controlled phases of the PREEMPT clinical program. Headache 2010; 50(6): 921-936

8. Goadsby PJ, de Coo IF, Silver N, et al. Noninvasive vagus nerve stimulation for the acute treatment of episodic and chronic cluster headache: a randomized, double-blind, shamcontrolled ACT2 study. Cephalalgia 2018; 38(5): 959-969.

9. Schoenen J, Vandersmissen B, Jeangette S, et al. Migraine prevention with a supraorbital transcutaneous stimulator: a randomized controlled trial. Neurology 2013; 80(8): 697-704

10. Lipton RB, Dodick DW, Silberstein SD, et al. Single-pulse transcranial magnetic stimulation for acute treatment of migraine with aura: a randomised, double-blind, parallel-group sham-controlled trial. Lancet Neurol 2010; 9(4): 373-380.

11. Cuadrado ML, Aledo-Serrano Á, Navarro P, et al. Short-term effects of greater occipital nerve blocks in chronic migraine: a double-blind, randomised, placebo-controlled clinical trial. Cephalalgia 2017; 37(9): 864-872.

12. Peres MF, Stiles MA, Siow HC, et al. Greater occipital nerve blockade for cluster headache. Cephalalgia 2002; 22(7): 520-522.

13. Amy R. Tso, Peter J. Goadsby. Anti-CGRP monoclonal antibodies: the next era of migraine prevention? Curr Treat Options Neurol 2017; 19(8): 27. 